\title{
Solution Processed Tungsten Oxide Interfacial Layer for Efficient Hole-Injection in Quantum Dot Light- Emitting Diodes
}

\author{
Xuyong Yang, Evren Mutlugun, Yongbiao Zhao, Yuan Gao, Kheng Swee Leck, \\ Yanyan Ma, Lin Ke, Swee Tiam Tan, Hilmi Volkan Demir, * and Xiao Wei Sun*
}

Light-emitting diodes (LEDs) based on colloidal quantum dots (QDs) are highly promising for the next generation of lighting and displays thanks to their narrow emission linewidth, ${ }^{[1-4]}$ tunable color emission spectral window across the visible to near-infrared range, ${ }^{[5-7]}$ and cost-effective fabrication techniques compatible with solution processed methods. ${ }^{\left[{ }^{8-12}\right]}$ Ever since the first demonstration of QDbased LEDs (QLEDs) 18 years ago, ${ }^{[13]}$ rapid progress has been made in the device performances owing to the technological development and accumulation of relevant knowledge in materials and device architectures, i.e., understanding the underlying device physics, ${ }^{[14-16]}$ designing more efficient

\author{
X. Y. Yang, Dr. E. Mutlugun, Dr. Y. B. Zhao, K. S. Leck, \\ Dr. S. T. Tan, Prof. H. V. Demir, Prof. X. W. Sun \\ Luminous! Center of Excellence for Semiconductor \\ Lighting and Displays, \\ School of Electrical and Electronic Engineering \\ Nanyang Technological University \\ Nanyang Avenue, Singapore, 639798, Singapore \\ E-mail: hvdemir@ntu.edu.sg; EXWSun@ntu.edu.sg \\ Dr. E. Mutlugun, Prof. H. V. Demir \\ Department of Electrical and Electronics Engineering \\ Department of Physics, UNAM - Institute of Materials Science \\ and Nanotechnology, \\ Bilkent University \\ Bilkent, Ankara, 0680o, Turkey \\ Y. Gao, Prof. H. V. Demir \\ School of Physical and Mathematical Sciences \\ Nanyang Technological University \\ Nanyang Avenue, Singapore, 639798, Singapore \\ Y. Y. Ma \\ State Key Lab of Luminescence Materials and Devices \\ Institute of Optical Communication Materials \\ South China University of Technology \\ Guangdong, 510641, China \\ Dr. L. Ke \\ Institute of Materials Research and Engineering \\ A* STAR (Agency for Science, Technology and Research) \\ 3 Research Link, Singapore, 117602, Singapore \\ Prof. X. W. Sun \\ South University of Science and Technology \\ 1088 Xue-Yuan Road, Shenzhen, Guangdong, 518055, China.
}

DOI: 10.1002/smll.201301199 device architectures, ${ }^{[17-20]}$ and improving quantum dot properties by adjusting their composition and structure. ${ }^{[21-23]}$ To date, QLEDs have emerged as an undeniable competitor to organic light-emitting diodes (OLEDs) for lighting and display applications. However, despite their apparent advantages, long-term stability of QLEDs is still a big concern for their practical applications.

Traditionally, polyethylene dioxythiophene:polystyrene sulfonate (PEDOT:PSS) is the most widely used buffer layer on an indium tin oxide (ITO) electrode for the fabrication of QLEDs. However, the aqueous PEDOT:PSS dispersion causes a side effect on the QLED stability due to its hygroscopic nature as well as acidic nature corroding the ITO electrode, resulting in the reduction of device lifetime. ${ }^{[24,25]}$ Furthermore, compared with the inorganic material-based devices, the organic interfacial buffer layers have inferior thermal stability. Efforts to replace PEDOT:PSS with metaloxides such as tungsten-, molybdenum-, nickel-, copper (I)-, rhenium-, or vanadium-oxides $\left(\mathrm{WO}_{3}, \mathrm{MoO}_{3}, \mathrm{NiO}, \mathrm{Cu}_{2} \mathrm{O}\right.$, $\mathrm{ReO}_{3}$, or $\mathrm{V}_{2} \mathrm{O}_{5}$ ) have gained significant importance in the recent years. ${ }^{[26-31]}$ In particular, highly n-doped $\mathrm{WO}_{3}$ and $\mathrm{MoO}_{3}$ exhibiting remarkably deep lying electronic states and efficient hole-injection into organic materials have been demonstrated. ${ }^{[32-35]}$ However, their unique electronic properties have so far been primarily achieved using thin films made by high-cost thermal evaporation under vacuum, which presents disadvantages due to the cost issues and incompatibility with roll-to-roll scalable manufacturing. Additionally, it has been demonstrated that metal-oxide nanoparticles (NPs) as the interfacial buffer layers are often more efficient as compared to their bulk counterparts. For example, $\mathrm{ZnO}$ nanoparticle films as the electron transporting layers (ETLs) prepared by a sol-gel method have been introduced, resulting in allsolution-processed QLEDs with the maximum brightness values of $31000 \mathrm{cdm}^{-2}, 68000 \mathrm{cdm}^{-2}$ and $4200 \mathrm{~cd} \mathrm{~m}^{-2}$ for red, green and blue devices, respectively, which are among the highest reported thus far. ${ }^{[8]}$ Recently, Meyer's group reported the preparation of solution-processed $\mathrm{MoO}_{3}$ nanoparticle films where the $\mathrm{MoO}_{3}$ was spin-coated on ITO from a suspension containing $\mathrm{MoO}_{3}$ nanoparticles and a block copolymer dispersing agent in xylene. ${ }^{[36]}$ However, the films require an extra $\mathrm{O}_{2}$-plasma treatment to remove the polymeric dispersing agent to facilitate hole injection in the device and the 
(a)

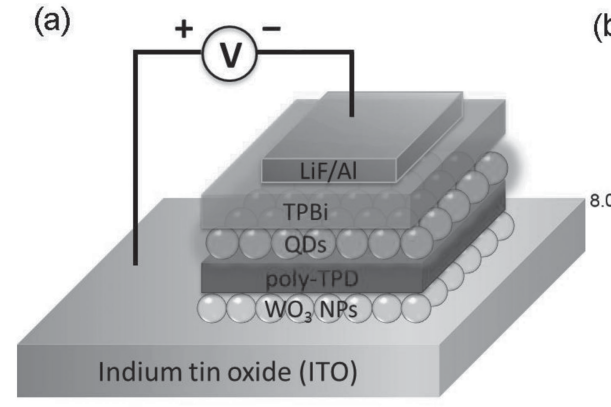

(b)
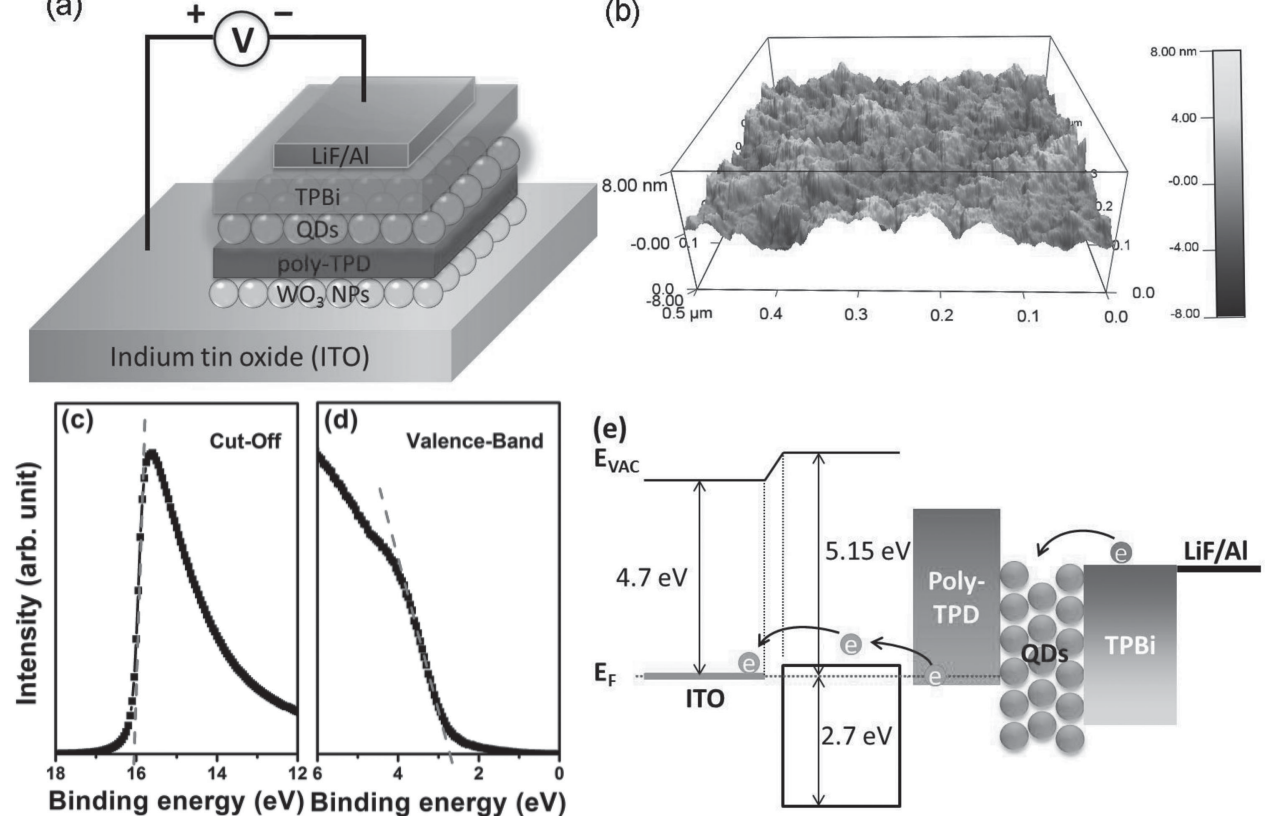

Figure 1. (a) Schematic of the device configuration of the QLEDs. (b) AFM image of WO 3 nanoparticle HTL formed on ITO substrate. UPS spectra of a $\mathrm{WO}_{3}$ nanoparticle film annealed at $100^{\circ} \mathrm{C}$ including (c) secondary-electron cutoff and (d) zoom-in section of the evolution of the density of states near the oxide valence band edge. (e) Energy level diagram of the $\mathrm{WO}_{3}$ nanoparticle-based QLEDs.

resulting surface of $\mathrm{MoO}_{3}$ nanoparticle film was relatively rough. Therefore, as such, in-depth studies focusing on solution-processed inorganic interfacial buffer layers is of critical importance for improving the QLED performance.

Here we report a highly efficient, stable QLED using solution-processed $\mathrm{WO}_{3}$ nanoparticles as the hole injection layer. The preparation of the $\mathrm{WO}_{3}$ nanoparticle layer described here is simple and cost-effective employing cheap and commercially available $\mathrm{WO}_{3}$ nanoparticles and ethanol as a solvent and utilizing a low-temperature process under ambient conditions (annealing temperature can be as low as $80^{\circ} \mathrm{C}$ and without requiring the $\mathrm{O}_{2}$-plasma treatment). This treatment temperature is much lower than that of PEDOT:PSS $\left(120-150{ }^{\circ} \mathrm{C}\right)$. At the same time, the overall performance for $\mathrm{WO}_{3}$ nanoparticle-based QLED is superior compared to that of the present PEDOT:PSS-based QLEDs using the same device architecture. The $\mathrm{WO}_{3}$ nanoparticle-based QLEDs with a maximum brightness of $30,006 \mathrm{~cd} / \mathrm{m}^{2}$, an external quantum efficiency (EQE) of $3.32 \%$, and a peak current efficiency of $10.75 \mathrm{~cd} \mathrm{~A}^{-1}$ have been achieved. Besides, the device lifetime has been also improved remarkably compared to that of PEDOT:PSS-based QLEDs, which marks as a further step towards the practical application of the QLED technology.

The standard structure of QLEDs is given as a multilayer structure of ITO/poly(3,4-ethylenedioxythiophene)poly(styrenesulfonate)(PEDOT:PSS)/Poly[ $N, N^{\prime}$ bis(4-butylphenyl)- $N, N^{\prime}$-bis(phenyl)benzidine] (poly-TPD)/ QDs/2, 2', 2"- (1, 3, 5-benzinetriyl)-tris(1-phenyl-1- $H$-benzimidazole) (TPBi)/LiF/Al). ${ }^{[17,22]}$ The $\mathrm{CdSe} / \mathrm{ZnS}$ core-shell structured QDs prepared according to a previous reported literature were used for the emissive layer. ${ }^{[3]}$ The poly-TPD and TPBi layers were chosen as the hole transport layer (HTL) and the electron transport layer (ETL), respectively.
In our case, the $\mathrm{WO}_{3}$ nanoparticle layer spin-coated on ITO was used to replace PEDOT:PSS as the HIL. The device structure is schematically presented in Figure 1a. The atomic force microscopy (AFM) image of the close-packed thin film of $\mathrm{WO}_{3}$ nanoparticles on ITO with an average particle size of $7 \mathrm{~nm}$ (annealed at $100{ }^{\circ} \mathrm{C}$ in the glove box) is shown in Figure 1b. The surface roughness (RMS) of the film made from a $2 \mathrm{wt} \%$ of $\mathrm{WO}_{3}$ ethanol solution is only $2.8 \mathrm{~nm}$, indicating that the nanoparticle film has a smooth surface. To confirm the electronic structure of the as-prepared $\mathrm{WO}_{3}$ nanoparticle film, the ultraviolet photoelectron spectroscopy (UPS) spectra including the magnified regions of the photoemission cut-off and valence band are given in Figures 1c and $1 \mathrm{~d}$. The photoemission onset is found at $16.05 \mathrm{eV}$ from the photoemission cut-off in Figure 1c. This corresponds to a work function (WF) of $5.15 \mathrm{eV}$ in agreement with the WF values of $\mathrm{WO}_{3}$ reported in literature, which can range from 4.7 to $6.4 \mathrm{eV}$ depending on the film preparation conditions. ${ }^{[37]}$ Figure 1d displays the zoom-in spectra of the density of states near the oxide valence band edge and the ionization energy (IE) of the $\mathrm{WO}_{3}$ film that is determined to be $2.7 \mathrm{eV}$ with respect to the Fermi level. According to the schematic energy level diagram of the device depicted in Figure 1e, it can be observed that the electrons can easily be injected from the Al to the QD layer. However, the case is quite different for the hole injection. ${ }^{24,34,36]}$ Owing to the deep lying electronic states of $\mathrm{WO}_{3}$ nanoparticle interface layer, efficient hole injection can be proceeded via electron extraction from the highest occupied molecular orbital (HOMO) level of poly-TPD into the conduction band of $\mathrm{WO}_{3}$ nanoparticles. For n-doped $\mathrm{WO}_{3}$ or $\mathrm{MoO}_{3}$, the hole injection from ITO to the organic semiconductors results from electron extraction from the highest occupied molecular orbital (HOMO) level 

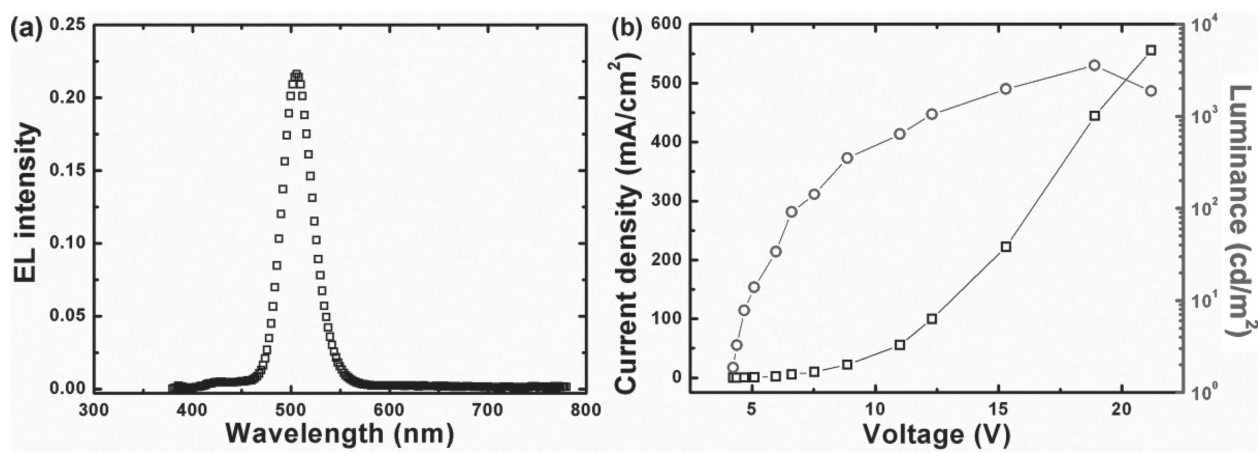

Figure 2. (a) EL spectrum and (b) current density-voltage and luminance-voltage characteristics of QLED.

of organic semiconductors through the $\mathrm{WO}_{3}$ or $\mathrm{MoO}_{3}$ conduction band, and then into ITO. For our case, owing to the deep lying electronic states of $\mathrm{WO}_{3}$ nanoparticle interface layer, the energy barrier for the injection of electrons from the HOMO level of to the conduction band of $\mathrm{WO}_{3}$ nanoparticles is quite small, and the efficient hole injection can be proceeded via electron extraction from the HOMO level of poly-TPD into the conduction band of $\mathrm{WO}_{3}$ nanoparticles.

Figure 2a presents the output performance of an optimized $\mathrm{WO}_{3}$ nanoparticle-based QLED. The device is fabricated with QDs of 4 monolayers-equivalent thickness ( $20 \mathrm{~nm})$

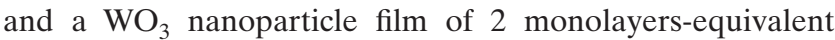
thickness $(\sim 14 \mathrm{~nm})$. The electroluminescence (EL) spectrum of the QLED was recorded at the bias voltage of $12 \mathrm{~V}$, showing a characteristic QD EL peak centered at $\sim 518 \mathrm{~nm}$ with a full-width-at-half-maximum (FWHM) of $\sim 30 \mathrm{~nm}$. It should be noted here that there is a weak emission in the blue-wavelength region due to the residual emission from poly-TPD. The emission from poly-TPD indicates the possible imbalance of the charge in the QD layer. The electrons on the lowest unoccupied molecular orbital (LUMO) of TPBi can jump over the $0.9 \mathrm{eV}$ energy barrier into the LUMO of poly-TPD through defects of the QD layer to combine with holes in the poly-TPD layer. This phenomenon can be observed in almost all PEDOT:PSS-based QLEDs. Figure $2 \mathrm{~b}$ shows the luminance and current density characteristics of the device as a function of the applied voltage. The luminance-current density-voltage characteristics show a low turn-on voltage of $3.6 \mathrm{~V}$ and a peak luminance of $3565 \mathrm{~cd} / \mathrm{m}^{2}$ at a current density of $444.44 \mathrm{~mA} / \mathrm{cm}^{2}$. The maximum EQE of $0.43 \%$ was obtained under the same current density.

To further improve the performance of $\mathrm{WO}_{3}$ nanoparticlebased QLEDs, we deposited a 2,2',2"-tris-(N-carbazolyl)triphenylamine (TCTA) electron-blocking layer (EBL) on the QD layer to suppress the excess electrons for improving the charge balance of device. The LUMO of TCTA is $2.4 \mathrm{eV}^{[38]}$ and the relatively high energy barrier of $0.8 \mathrm{eV}$ between the LUMO of TCTA and that of TPBi can block electrons to reach the poly-TPD layer in the device effectively. Figure $3 \mathrm{a}$ shows the EL spectra for the device with TCTA utilized as the EBL under different driving currents. It can be observed that the blue emission of poly-TPD is hardly found even in the case of a high driving current, as electrons from TPBi are completely blocked by the TCTA EBL before jumping into the LUMO of poly-TPD. This is another advantage of using TCTA because the blue emission from poly-TPD degrades the color purity. Compared with the original QLEDs without the TCTA used as the EBL, the performance of QLED with TCTA is increased significantly, showing the maximum luminance, EQE and current efficiency values of $30006 \mathrm{~cd} \mathrm{~m}^{-2}$ and $3.32 \%$, respectively. To the best of our knowledge, the EQE of $3.32 \%$ is much higher than the previously reported values for green QLEDs with normal architecture (the recently reported green QLEDs using the inverted architecture reached an EQE level of $5.8 \%){ }^{[17]}$ A corresponding image of the QLED output was recorded at the luminance of $300 \mathrm{~cd} / \mathrm{m}^{2}$, which displays bright and saturated green emission (Figure $3 b$ ). Figures $3 c$ and $3 d$ show a comparison of the luminance-current density $(L-J)$ and efficiency-current density $(E-J)$ characteristics of the $\mathrm{WO}_{3}$ nanoparticle-based QLED and an optimized PEDOT:PSS-based QLED with the same structure. As can be seen, the two devices show a similar trend in the $L-J$ and $E-J$ characteristics, as the driving current is increased. Table $\mathbf{1}$ summarizes the key figures of the two QLEDs. The maximum brightness, EQE, current efficiency and efficacy for the $\mathrm{WO}_{3}$ nanoparticle-based QLED are superior. Also, the turn-on voltage of the $\mathrm{WO}_{3}$ nanoparticle-based QLED decreases to $3.8 \mathrm{~V}$, which is lower than that observed for the PEDOT:PSS-based device (4.2 V). Moreover, it can be noted that the $\mathrm{WO}_{3}$ nanoparticle-based QLED still works well even under the current of $50 \mathrm{~mA}$, while the PEDOT:PSS-based QLED has burned down when the driving current is just $40 \mathrm{~mA}$, which indicates the better device stability for the $\mathrm{WO}_{3}$ nanoparticle-based QLED.

Figure 4 shows the operating stability of the resulting unencapsulated $\mathrm{WO}_{3}$ nanoparticle-based QLED in comparison with the optimized PEDOT:PSS-based QLED. Under a continuous current driving condition corresponding to an initial luminance of $1000 \mathrm{~cd} / \mathrm{m}^{2}$, it can be clearly observed that using $\mathrm{WO}_{3}$ nanoparticles to replace PEDOT:PSS as the HILs in QLEDs can drastically improve device stability. The unencapsulated $\mathrm{WO}_{3}$ nanoparticle-based QLED displays a half-lifetime of ca. $6530 \mathrm{~s}$, showing an approximately two-fold lifetime enhancement as compared to that of PEDOT:PSSbased QLED. The significant improvement in the device stability can be attributed to the fact that $\mathrm{WO}_{3}$ nanoparticles are highly stable when compared to organic materials and can therefore act as a protection layer for organic materials. 

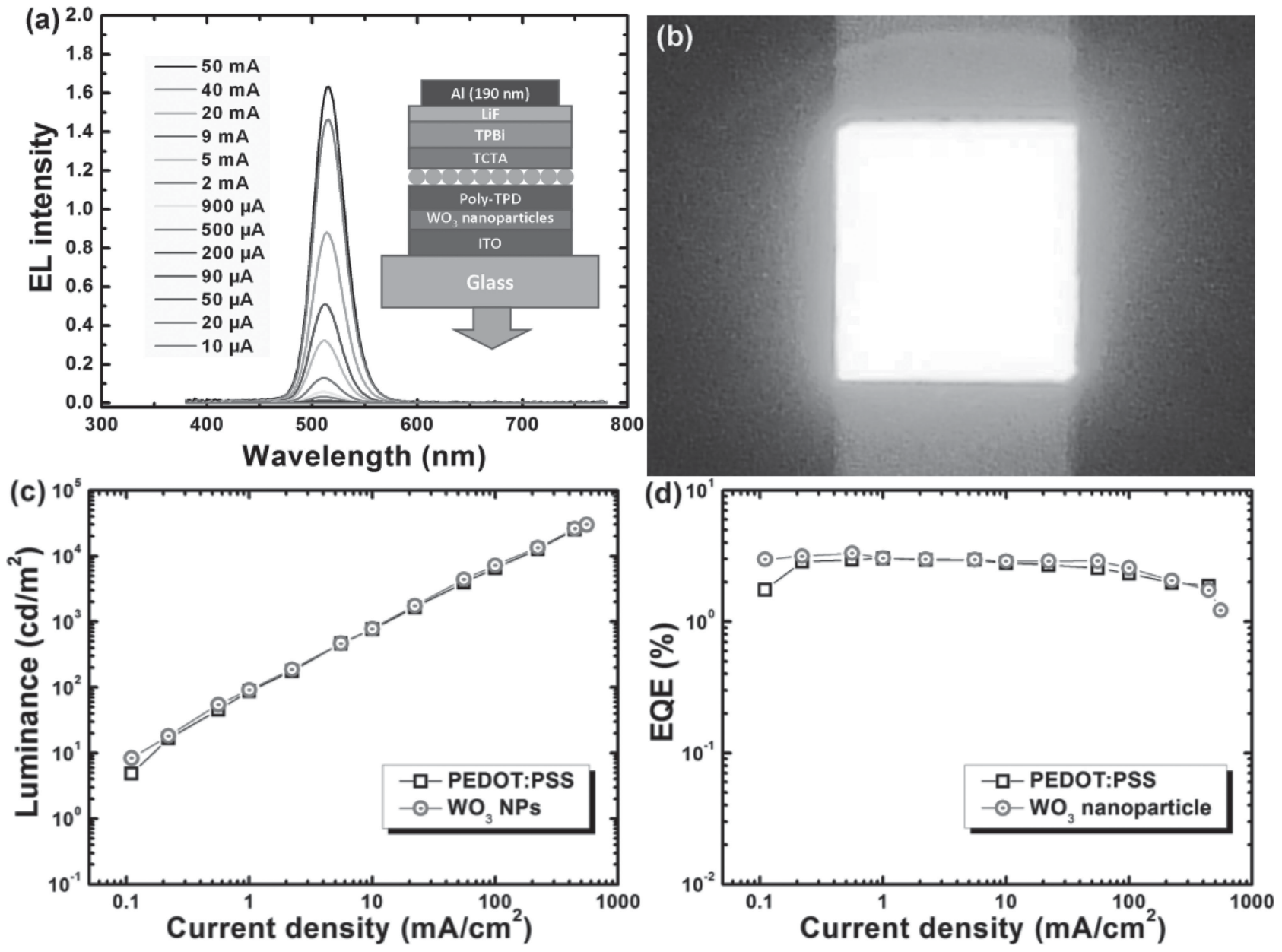

Figure 3. (a) EL spectra of the device at different injection currents (inset shows the schematic cross section of the device structure used in this study). (b) The photograph of a QLED under operation with a pixel size of $3 \mathrm{~mm} \times 3 \mathrm{~mm}$. (c) Luminance (cd $\mathrm{m}^{-2}$ ) and (d) external quantum efficiency (\%) of QLEDs using PEDOT:PSS and $\mathrm{WO}_{3}$ nanoparticles as the HTLS as a function of current density $\left(\mathrm{mA} \mathrm{cm}^{-2}\right)$.

In summary, we have demonstrated an efficient QLED using solution-processed $\mathrm{WO}_{3}$ nanoparticle film instead of using PEDOT:PSS as the anode interfacial buffer layer. The $\mathrm{WO}_{3}$ nanoparticle-based QLEDs show enhanced performance as compared to the PEDOT:PSS-based QLEDs. The EQE of $3.32 \%$ reported here is the highest value for green QLEDs using a non-inverted structure and the brightness of $30006 \mathrm{~cd} / \mathrm{m}^{2}$ matches that of the best QLEDs with organic materials used as HTLs/HILs. Moreover, with the incorporation of the $\mathrm{WO}_{3}$ nanoparticles, the unencapsulated device exhibits a significant improvement in the device stability and the lifetime is increased by approximately two-folds at an initial brightness of $1000 \mathrm{~cd} / \mathrm{m}^{2}$ as compared to that of PEDOT:PSS-based QLED. Meanwhile, since the solutionprocessed preparation method of $\mathrm{WO}_{3}$ nanoparticle film used in our work is very simple and can be achieved at low annealing temperatures, it is suitable for application in flexible devices where the flexible substrates such as plastics often cannot withstand high annealing temperatures. These results indicate that $\mathrm{WO}_{3}$ nanoparticles are promising solution-processed buffer layer materials and offer a practicable platform for the realization of high-performance, stable and large-area commercial QLEDs using a low-cost manufacturing process.

Table 1. List of figure-of-merits to compare the device performance using $\mathrm{WO}_{3}$ nanoparticles (A) and PEDOT:PSS (B) as HILs.

\begin{tabular}{|c|c|c|c|c|c|c|}
\hline Device & $\begin{array}{c}\mathrm{V}_{\text {turn-on }} \\
{[\mathrm{V}]}\end{array}$ & $\begin{array}{l}\text { Max. Luminance } \\
{[\mathrm{cd} / \mathrm{m} 2]}\end{array}$ & $\begin{array}{l}\text { Max. EQE } \\
{[\%]}\end{array}$ & $\begin{array}{l}\text { Max. Current efficiency } \\
{[\mathrm{cd} / \mathrm{A}]}\end{array}$ & $\begin{array}{l}\text { Max. Efficacy } \\
{[\mathrm{lm} / \mathrm{W}]}\end{array}$ & $\begin{array}{l}\text { Lifetime } \\
\quad[\mathrm{s}]\end{array}$ \\
\hline A & 3.8 & 30006 (@50mA) & 3.32 & 9.75 & 6.8 & 6530 \\
\hline$B$ & 4.2 & 25202 (@40 mA) & 3.02 & 8.74 & 6.0 & 3130 \\
\hline
\end{tabular}




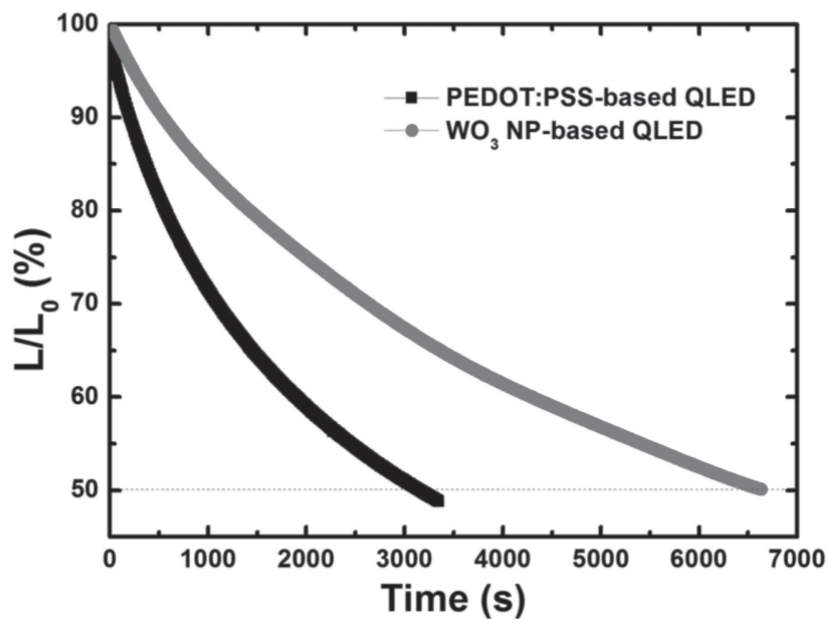

Figure 4. Operational lifetime characteristics of the resulting $\mathrm{WO}_{3}$ nanoparticle based QLED and the optimized PEDOT:PSS based QLED. The luminance of these QLEDs was measured without using encapsulation and during the continuous operation at a constant current density corresponding to an initial luminance of $1000 \mathrm{~cd} / \mathrm{m}^{2}$. Both types of QLEDs were fabricated using the same device architecture.

\section{Experimental Details}

Synthesis of CdSe-ZnS Core-Shell QDs: Green-emitting CdSeZnS QDs with a chemical-composition gradient were prepared according to a modified method reported in the literature. ${ }^{[3]}$ For a typical preparation of green-emitting QDs, $0.1 \mathrm{mmol}$ of cadmium oxide (CdO), $4 \mathrm{mmol}$ of zinc acetate $\left(\mathrm{Zn}(\text { Acet })_{2}\right), 5 \mathrm{~mL}$ of oleic acid $(O A)$ were loaded in a $50 \mathrm{~mL} 3$-neck flask, heated to $150{ }^{\circ} \mathrm{C}$ under vacuum to form cadmium oleate $\left(\mathrm{Cd}(\mathrm{OA})_{2}\right)$ and zinc oleate $\left(\mathrm{Zn}(\mathrm{OA})_{2}\right)$. Then $20 \mathrm{~mL}$ of 1 -octadecene (1-ODE) was added to the reaction flask and the reactor was then filled with nitrogen and heated up to $300^{\circ} \mathrm{C}$. At the elevated temperature, $1.6 \mathrm{~mL}$ of tri- $\mathrm{n}$ octylphosphine (TOP) dissolving $0.15 \mathrm{mmol}$ of selenium (Se) and $4 \mathrm{mmol}$ of sulphur (S) was injected into the flask swiftly, and the reaction mixture were maintained at $300{ }^{\circ} \mathrm{C}$ for $10 \mathrm{~min}$ for the QD growth. To purify the synthesized QDs, the reaction mixture was cooled down to room temperature, and the QDs were extracted by the addition of acetone and methanol, followed by centrifugation. The CdSe-ZnS core-shell QDs were readily dispersed in toluene.

Fabrication of QLED Devices: The patterned ITO substrates were cleaned by sonication sequentially in detergent, de-ionized water, acetone, and isopropyl alcohol. The $\mathrm{WO}_{3}$ anode buffer layer was spin-coated on the $\mathrm{O}_{2}$-plasma treated ITO substrate from diluted $1.25 \mathrm{wt} \%$ of $\mathrm{WO}_{3}$ enthanol solution at $5000 \mathrm{rpm}$ for $60 \mathrm{~s}$ and annealed at $80-110^{\circ} \mathrm{C}$ for $30 \mathrm{~min}$. The $2 \mathrm{wt} \%$ of poly-TPD $(50 \mathrm{~nm})$ in chlorobenzene was also spin-coated on the $\mathrm{WO}_{3}$ layer at $4000 \mathrm{rpm}$ for $60 \mathrm{~s}$, followed by thermal annealing at $150{ }^{\circ} \mathrm{C}$ for $30 \mathrm{~min}$ in a nitrogen glove box. The QD layer was then deposited on the ITO/ $\mathrm{WO}_{3} /$ poly-TPD layer by spin-coating the $\mathrm{QD}$ dispersion (QDs were dispersed in toluene with $15 \mathrm{mg} / \mathrm{mL}$ ) at a rate of $1000-$ $4000 \mathrm{rpm}$ for $60 \mathrm{~s}$, and cured at $90{ }^{\circ} \mathrm{C}$ under $\mathrm{N}_{2}$ atmosphere for $30 \mathrm{~min}$. The TPBi $(35 \mathrm{~nm}), \operatorname{LiF}(0.5 \mathrm{~nm})$, and Al (190 nm) layers were thermally deposited under a base pressure of $\sim 2 \times 10^{-4} \mathrm{~Pa}$. $\mathrm{WO}_{3}$ dispersion in enthanol (2.5 wt\%) was purchased from Nanograde $\mathrm{GmbH}$ (product no. 4035). For PEDOT:PSS based QLEDs, we followed the same procedure and only the $\mathrm{WO}_{3}$ layer was replaced by PEDOT:PSS layer $(40 \mathrm{~nm})$ which was spun on the ITO substrate at $4000 \mathrm{rpm}$ for $60 \mathrm{~s}$ and annealed at $150^{\circ} \mathrm{C}$ for $30 \mathrm{~min}$.

Instrumentation: AFM (Cypher AFM, Asylum Research) was used to image the $\mathrm{WO}_{3}$ nanoparticle film. UPS was performed using X-Ray Photoelectron spectroscopy (XPS) (VG Escalab 220i $\mathrm{XL})$ with a He I (21.2 eV) gas discharge lamp. The electroluminescence (EL) spectra of the fabricated devices were measured using a PR650 Spectra Scan spectrometer, while the luminance-current density-voltage (L-J-V) characteristics were obtained simultaneously, by connecting the spectrometer to a programmable Keithley 236 source measurement unit. All measurements were carried out at room temperature under ambient conditions.

\section{Acknowledgments}

The authors would like to thank the financial support from Singapore National Research Foundation under NRF-RF-2009-09 and NRF-CRP-6-2010-02 and the Science and Engineering Research Council, Agency for Science, Technology and Research ( $A$ *STAR) of Singapore (project Nos. 0921010057 and 112120 2009). The work is also supported by the National Natural Science Foundation of China (NSFC) (project Nos. 61006037 and 61076015).

[1] Y. Shirasaki, G. J. Supran, M. G. Bawendi, V. Bulovic, Nat. Photonics 2013, 7, 13-23.

[2] K. S. Cho, E. K. Lee, W. J. Joo, E. Jang, T. H. Kim, S. J. Lee, S. J. Kwon, J. Y. Han, B. K. Kim, B. L. Choi1, J. M. Kim, Nat. Photonics 2009, 3, 341-345.

[3] W. K. Bae, J. Kwak, J. Lim, D. Lee, M. K. Nam, K. Char, C. Lee, S. Lee, Nano Lett. 2010, 10, 2368-2373.

[4] V. Wood, V. Bulović, Nano Rev. 2010, 1, 5202-5208.

[5] X. Yang, D. Zhao, K. S. Leck, S. T. Tan, Y. X. Tang, J. Zhao, H. V. Demir, X. W. Sun, Adv. Mater. 2012, 24, 4180-4185.

[6] P. O. Anikeeva, J. E. Halpert, M. G. Bawendi, V. Bulović, Nano Lett. 2009, 9, 2532-2536.

[7] L. Sun, J. J. Choi, D. Stachnik, A. C. Bartnik, B.-R. Hyun, G. G. Malliaras, T. Hanrath, F. W. Wise, Nat. Nanotechnol. 2012, 7, 369-373.

[8] L. Qian, Y. Zheng, J. Xue, P. H. Holloway, Nat. Photonics 2011, 5, 543-548.

[9] Y. Zhang, C. Xie, H. Su, J. Liu, S. Pickering, Y. Wang, W. W. Yu, J. Wang, Y. Wang, J. I. Hahm, N. Dellas, S. E. Mohney, J. Xu, Nano Lett. 2011, 11, 329-332.

[10] M. A. Schreuder, K. Xiao, I. N. Ivanov, S. M. Weiss, S. J. Rosenthal, Nano Lett. 2010, 10, 573-576.

[11] Z. Tan, F. Zhang, T. Zhu, J. Xu, A. Y. Wang, J. D. Dixon, L. Li, Q. Zhang, S. E. Mohney, Nano Lett. 2007, 7, 3803-3807.

[12] Z. Tan, Y. Zhang, C. Xie, H. Su, J. Liu, C. Zhang, N. Dellas, S. E. Mohney, Y. Wang, J. Wang, J. Xu, Adv. Mater. 2011, 23, 3553-3558.

[13] V. L. Colvin, M. C. Schlamp, A. P. Alivisatos, Nature 1994, 370, 354-357.

[14] D. Bozyigit, O. Yarema, V. Wood, Adv. Funct. Mater. 2013, doi: 10.1002/adfm.201203191.

[15] X. Yang, Y. Divayana, D. Zhao, K. S. Leck, F. Lu, S. T. Tan, A. P. Abiyasa, Y. Zhao, H. V. Demir, X. W. Sun, Appl. Phys. Lett. 201 2, 101, 233110-233113. 
[16] M. L. Mastronardi, E. J. Henderson, D. P. Puzzo, Y. Chang, Z. B. Wang, M. G. Helander, J. Jeong, N. P. Kherani, Z. Lu, G. A. Ozin, Small 2012, 12, 3647-3654.

[17] J. Kwak, W. K. Bae, D. Lee, I. Park, J. Lim, M. Park, H. Cho, H. Woo, D. Y. Yoon, K. Char, S. Lee, C. Lee, Nano Lett. 2012, 12, 2362-2366.

[18] V. Wood, M. J. Panzer, J. M. Caruge, J. E. Halpert, M. G. Bawendi, V. Bulovic', Nano Lett. 2010, 10, 24-29.

[19] E. M. Likovich, R. Jaramillo, K. J. Russell, S. Ramanathan, V. Narayanamurti, Adv. Mater. 2011, 23, 4521-4525.

[20] Q. Sun, G. Subramanyam, L. Dai, M. Check, A. Campbell, R. Naik, J. Grote, Y. Wang, ACS Nano 2009, 3, 737-743.

[21] B. N. Pal, Y. Ghosh, S. Brovelli, R. Laocharoensuk, V. I. Klimov, J. A. Hollingsworth, H. Htoon, Nano Lett. 2012, 12, 331-336.

[22] W. K. Bae, J. Kwak, J. W. Park, K. Char, C. Lee, S. Lee, Adv. Mater. 2009, 21, 1690-1694.

[23] B. Chen, H. Zhong, W. Zhang, Z. Tan, Y. Li, C. Yu, T. Zhai, Y. Bando, S. Yang, B. Zou, Adv. Fun. Mater. 2012, 22, 2011-2088.

[24] S. Murase, Y. Yang, Adv. Mater. 2012, 24, 2459-2462.

[25] J. J. Jasieniak, J. Seifter, J. Jo, T. Mates, A. J. Heeger, Adv. Funct. Mater. 2012, 22, 2594-2605

[26] M. T. Greiner, M. G. Helander, W.-M. Tang, Z.-B. Wang, J. Qiu, Z.-H. Lu, Nat. Mater. 2012, 11, 76-81.

[27] J. M. Caruge, J. E. Halpert, V. Wood, V. Bulović, M. G. Bawendi, Nat. Photonics 2008, 2, 247-250.
[28] Z. Tan, L. Li, C. Cui, Y. Ding, Q. Xu, S. Li, D. Qian, Y. Li, J. Phys. Chem. C 2012, 116, 18626-18632.

[29] H. M. Wei, H. B. Gong, L. Chen, M. Zi, B. Q. Cao, J. Phys. Chem. C 2012, 116, 10510-10515.

[30] J. Meyer, K. Zilberberg, T. Ried, A. Kahn, J. Appl. Phys. 2011, 110, 033710-033714.

[31] J.-H. Lee, D.-S. Leema, H.-J. Kim, J.-J. Kim, Appl. Phys. Lett. 2009, 94, 123306-123308.

[32] M. Sessolo, H. J. Bolink, Adv. Mater. 2011, 23, 1829-71845.

[33] R. Acharya, X. A. Cao, Appl. Phys. Lett. 2012, 101, 053306-053309.

[34] M. Kröger, S. Hamwi, J. Meyer, T. Riedl, W. Kowalsky, A. Kahn, Appl. Phys. Lett. 2009, 95, 123301-123303.

[35] Z. B. Wang, M. G. Helander, J. Qiu, D. P. Puzzo, M. T. Greiner, Z. M. Hudson, S. Wang, Z. W. Liu, Z. H. Lu, Nat. Photonics 2011, 5, 753-757.

[36] J. Meyer, R. Khalandovsky, P. Görrn, A. Kahn, Adv. Mater. 2011, 23, 70-73.

[37] S. Han, W. S. Shin, M. Seo, D. Gupta, S.-J. Moon, S. Yoo, Org. Electron. 2009, 10, 791-797.

[38] J. Y. Lee, M.-S. Gong, S. Ryu, G.-k. Chang, H. J. Chang, J. Appl. Phys. 2008, 103, 054502-054505.
Received: April 19, 2013 Revised: May 27, 2013 Published online: 